\title{
SCOPE: A Coronagraph for Operational Space Weather Prediction Phase A/B1 Design and Breadboarding
}

Kevin F. Middleton, Heiko Anwand, Volker Bothmer, Jackie A. Davies, Klaus Ergenzinger, Chris J. Eyles, Robert Hardie, Marie-Laure Hellin, Johannes Hinrichs, Philipp Huke, Piers Jiggens, Volker Kirschner, Emmanuel Mazy, Thomas McCarron, Bogdan Nicula, Lars Stopfkuchen, S. James Tappin, Ian A. J. Tosh, Nick R. Waltham, Matthew J. West

kevin.middleton@stfc.ac.uk
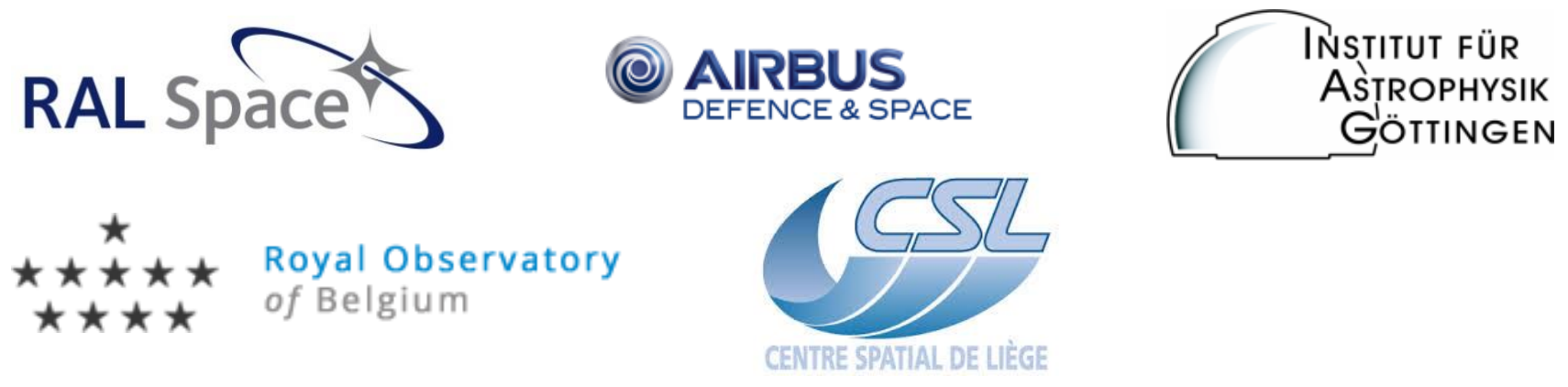
- Accurate prediction of solar wind phenomena, especially coronal mass ejections (CMEs), is becoming increasingly important

- A coronagraph provides earliest definitive evidence of CME eruption

- We are currently reliant on science instruments, some of them ageing (e.g. LASCO/SOHO, STEREO)

- SCOPE will be a compact, lightweight, low power, robust and durable coronagraph, optimised for operational space weather services

- ESA GSTP-funded conceptual design study with breadboarding of critical technologies
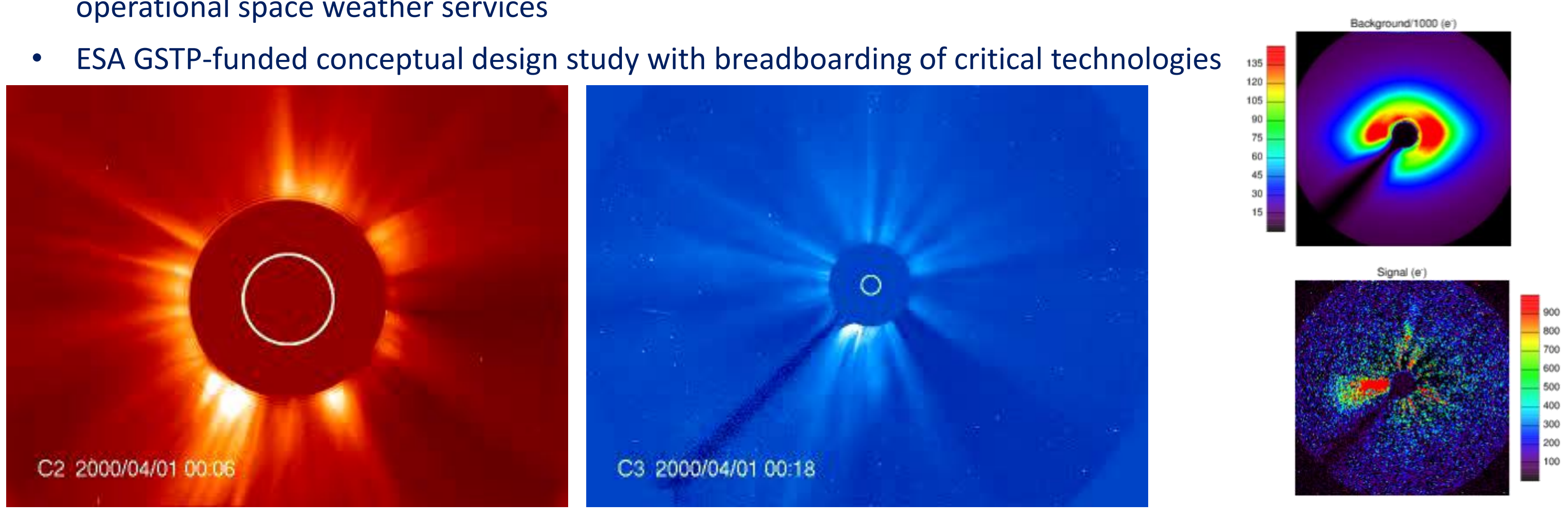


\begin{tabular}{|c|c|c|c|}
\hline Parameter & Values & Units & Notes \\
\hline Aperture diameter & 25 & $\mathrm{~mm}$ & \\
\hline Field of view & $3-30$ & $\mathrm{R}$ & Solar radii \\
\hline Physical detector format & $2048 \times 2048$ & pixels & Bin to $1024 \times 1024$ or $512 \times 512$ \\
\hline $\begin{array}{l}\text { Pixel size (physical detector } \\
\text { pixel) }\end{array}$ & 15 & $\mu \mathrm{m}$ & Bin to $30 \mu \mathrm{m}$ or $60 \mu \mathrm{m}$ \\
\hline Overall length & 710 & $\mathrm{~mm}$ & Front of baffle tube to detector \\
\hline Exposure time & 1 & $\mathrm{~s}$ & \\
\hline No. exposures & 6 & - & $\begin{array}{l}6 \text { exposures are combined into one } \\
\text { image }\end{array}$ \\
\hline $\begin{array}{l}\text { Effective total time to take one } \\
\text { image }\end{array}$ & 11 & $\mathrm{~s}$ & $\begin{array}{l}\text { Total for } 3 \text { exposures, including read- } \\
\text { out time }\end{array}$ \\
\hline $\begin{array}{l}\text { Fraction of used full-well } \\
\text { capacity }\end{array}$ & $90 \%$ & - & $\begin{array}{l}\text { Per exposure (assumes } 200 \text { ke- full- } \\
\text { well capacity) }\end{array}$ \\
\hline Limiting $\mathrm{R}$ for vignetting & 5.6 & $\mathrm{R}$ & $\begin{array}{l}\text { FOV at which vignetting no longer } \\
\text { degrades image quality }\end{array}$ \\
\hline SNR at $22 \mathrm{R}$ & 7 & - & $\begin{array}{l}\text { SNR per pixel for } 1 \text { image (3 combined } \\
\text { exposures) }\end{array}$ \\
\hline SNR at $30 \mathrm{R}$ & 3 & - & $\begin{array}{l}\text { SNR per pixel for } 1 \text { image ( } 3 \text { combined } \\
\text { exposures) }\end{array}$ \\
\hline
\end{tabular}




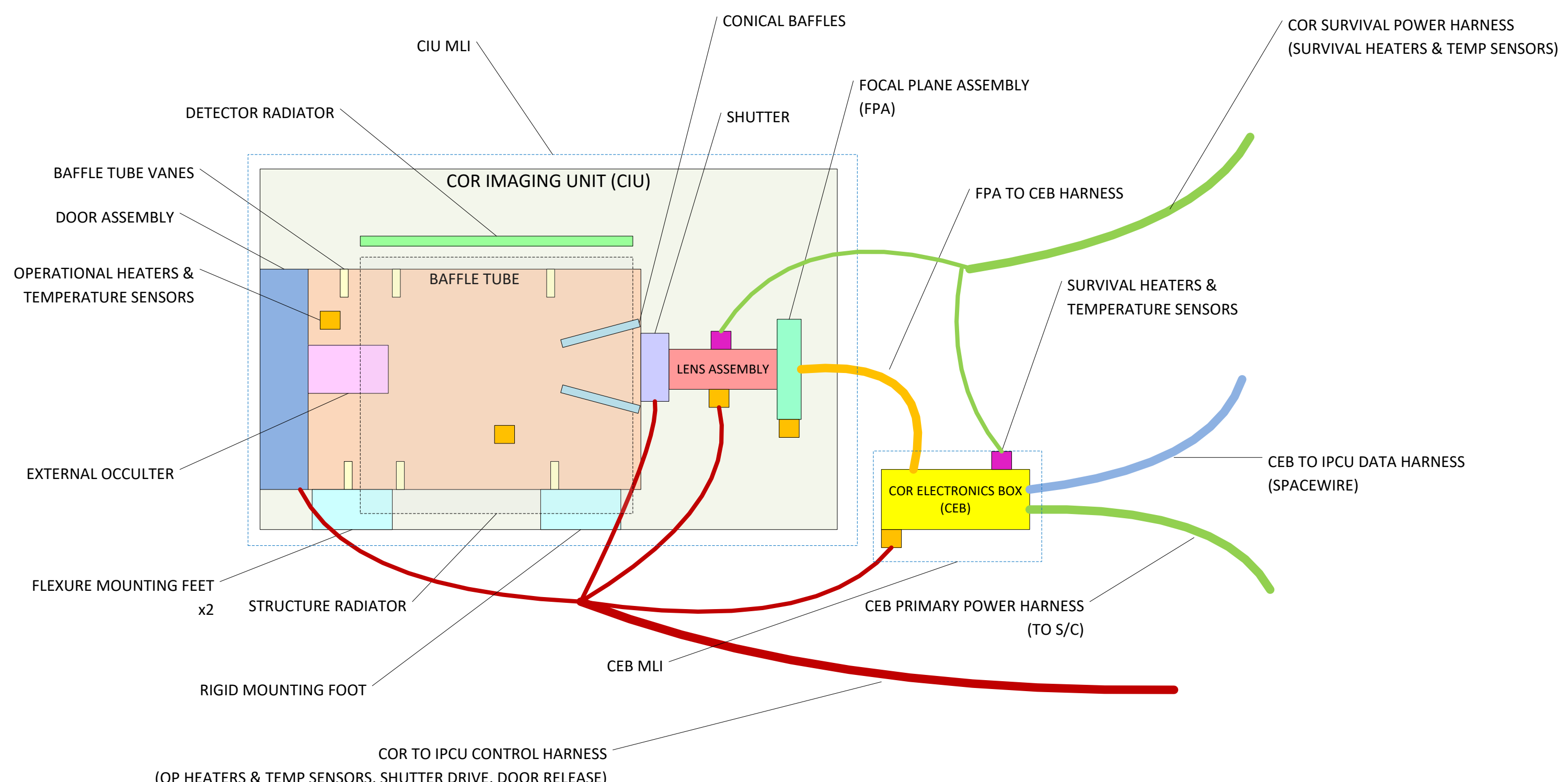




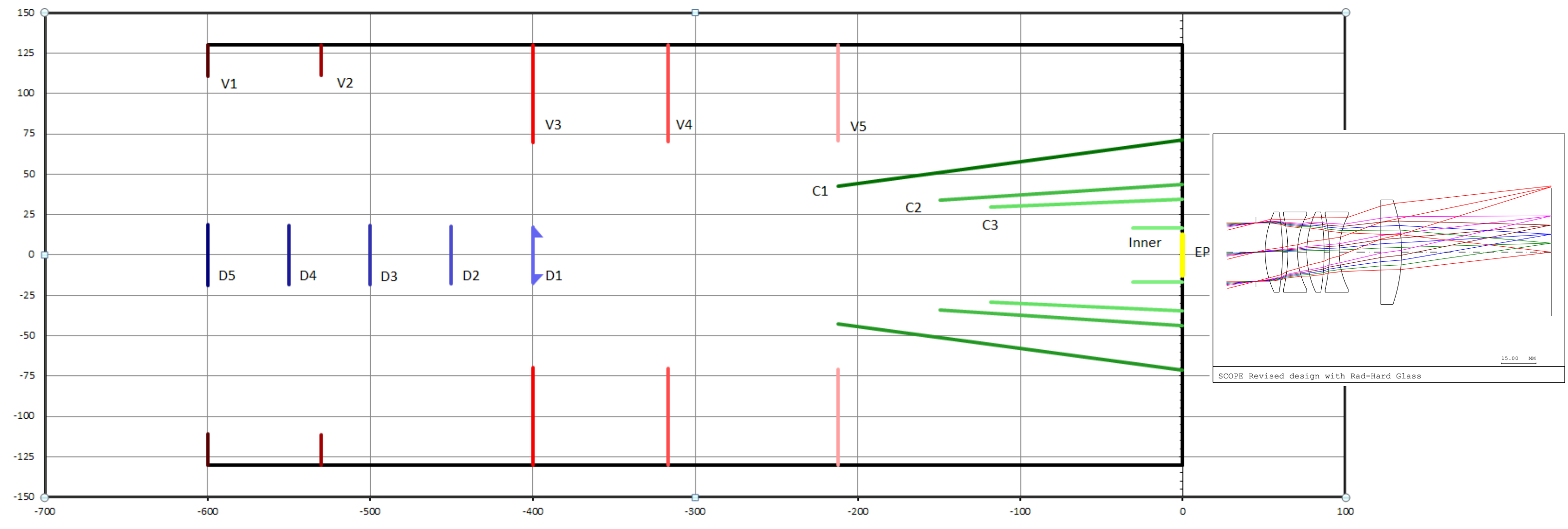




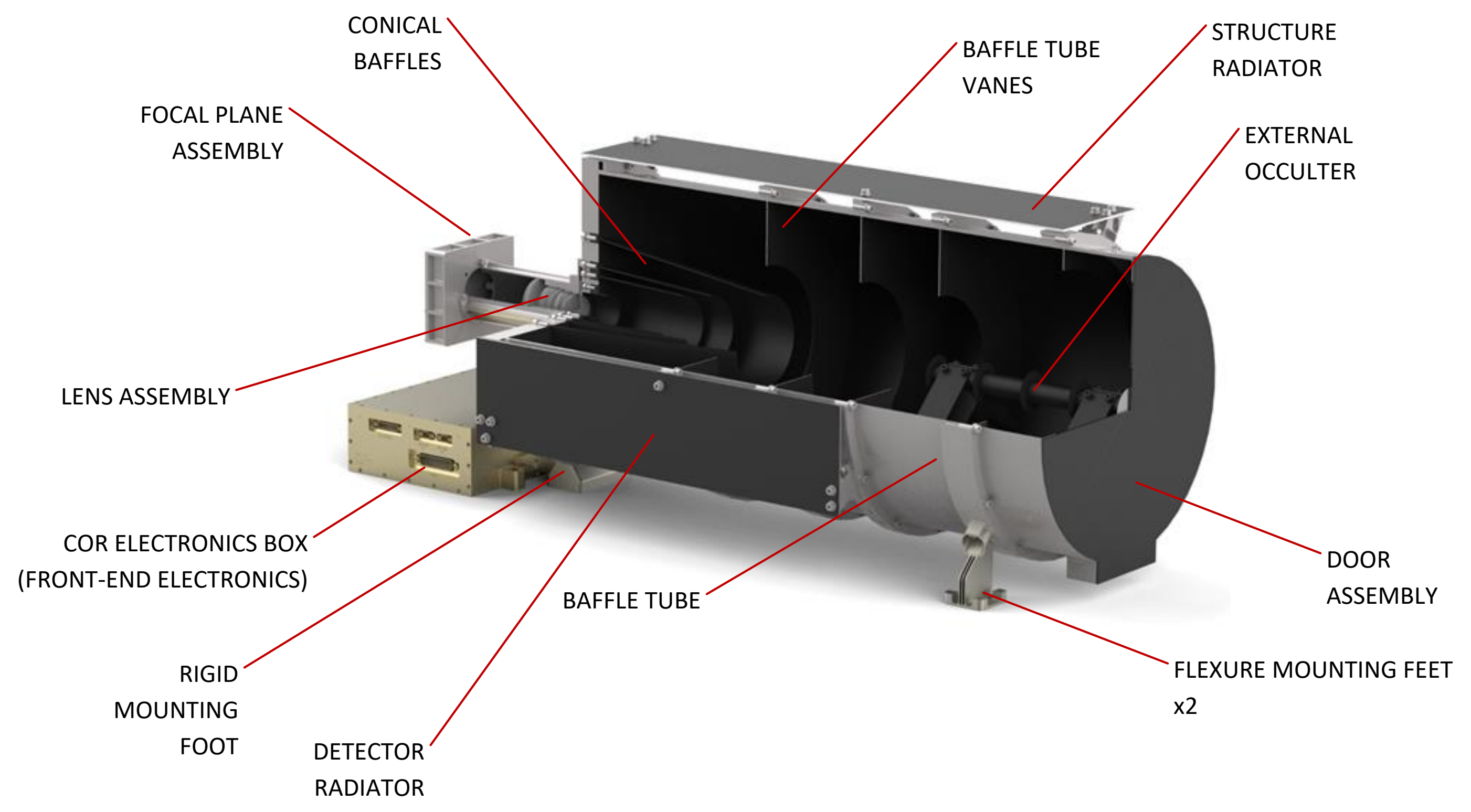



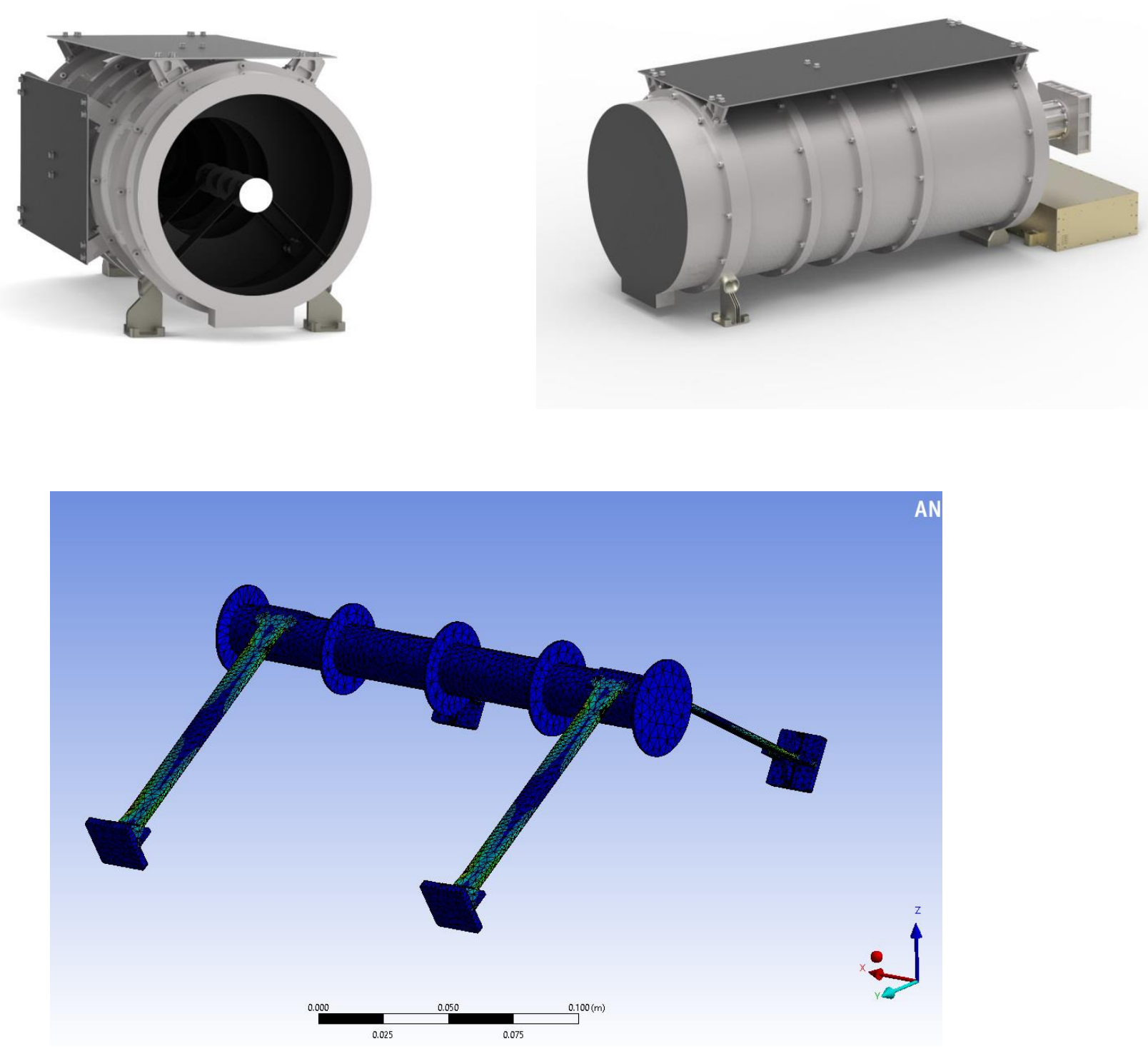

$\Rightarrow$

Attribute: Temperature

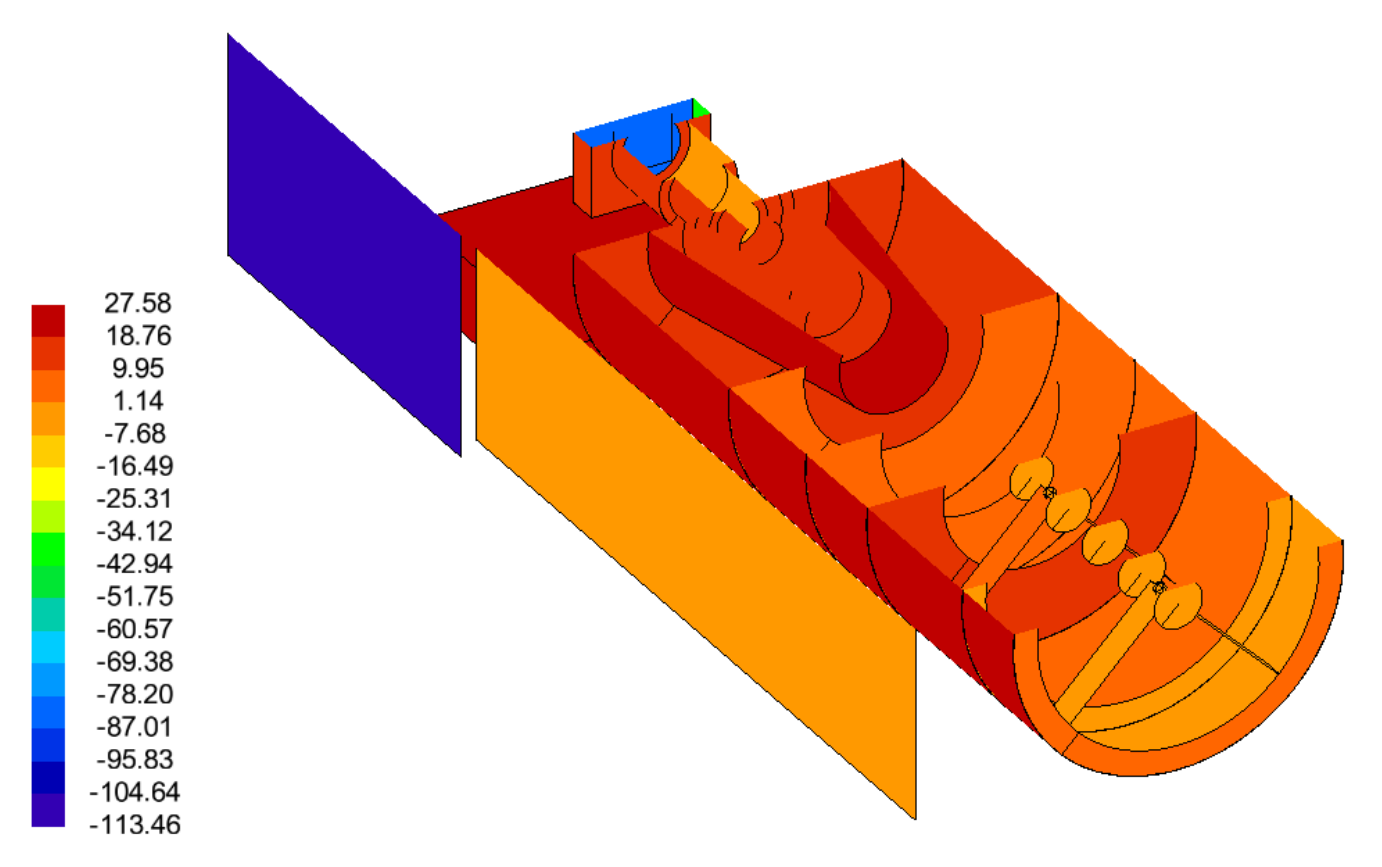

Thermo-optical Properties
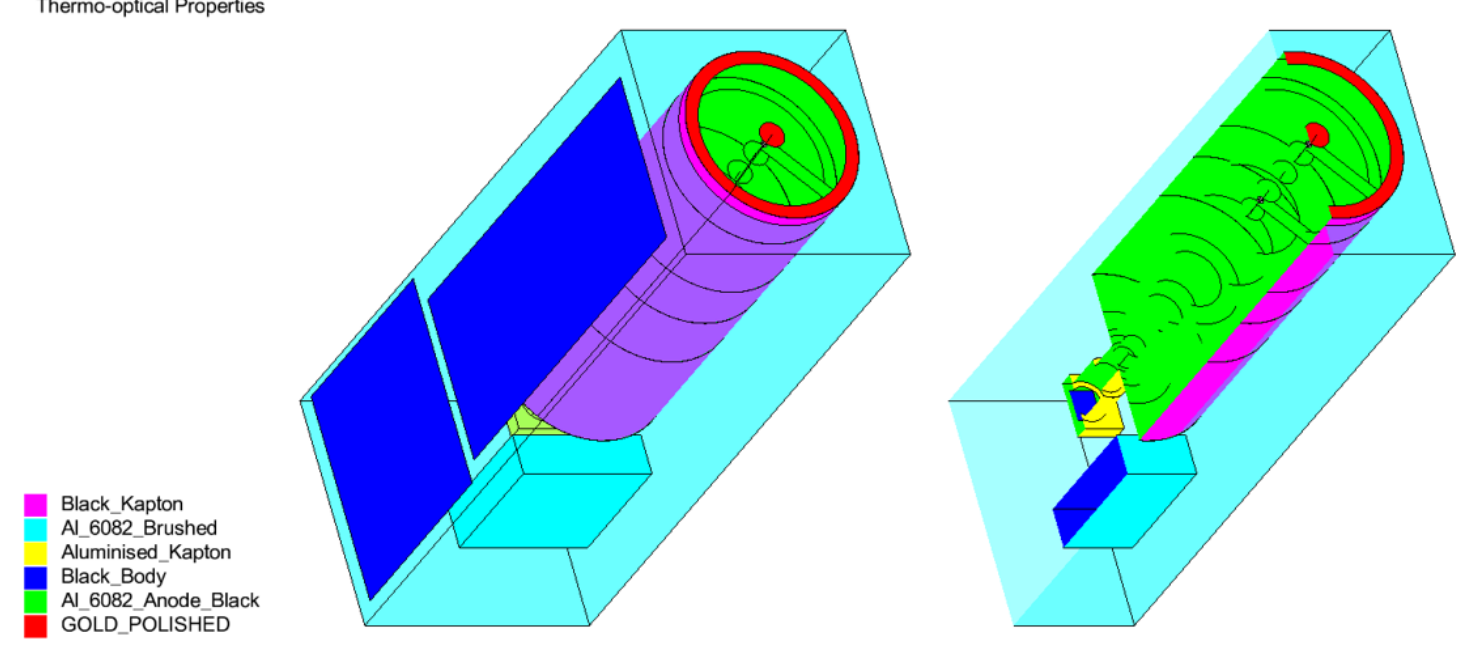
- Detector front-end electronics (FEE) and data handling unit $(\mathrm{DHU})$ in separate enclosures with separate power supplies

- $\quad$ Connected by SpaceWire interface

- $\mathrm{FEE}$

- Drives detector (e2v CCD230)

- Digitises image data

- Based on SDO AIA/HMI and GOES-R SUIV camera electronics

- DHU

- Based on LEON2-FT

- Local image storage

- Basic image processing

- Onboard CME identification (TBC)

- Mechanism and heater control

- Onboard software

- Image calibration, cleaning and compression

- Event recognition

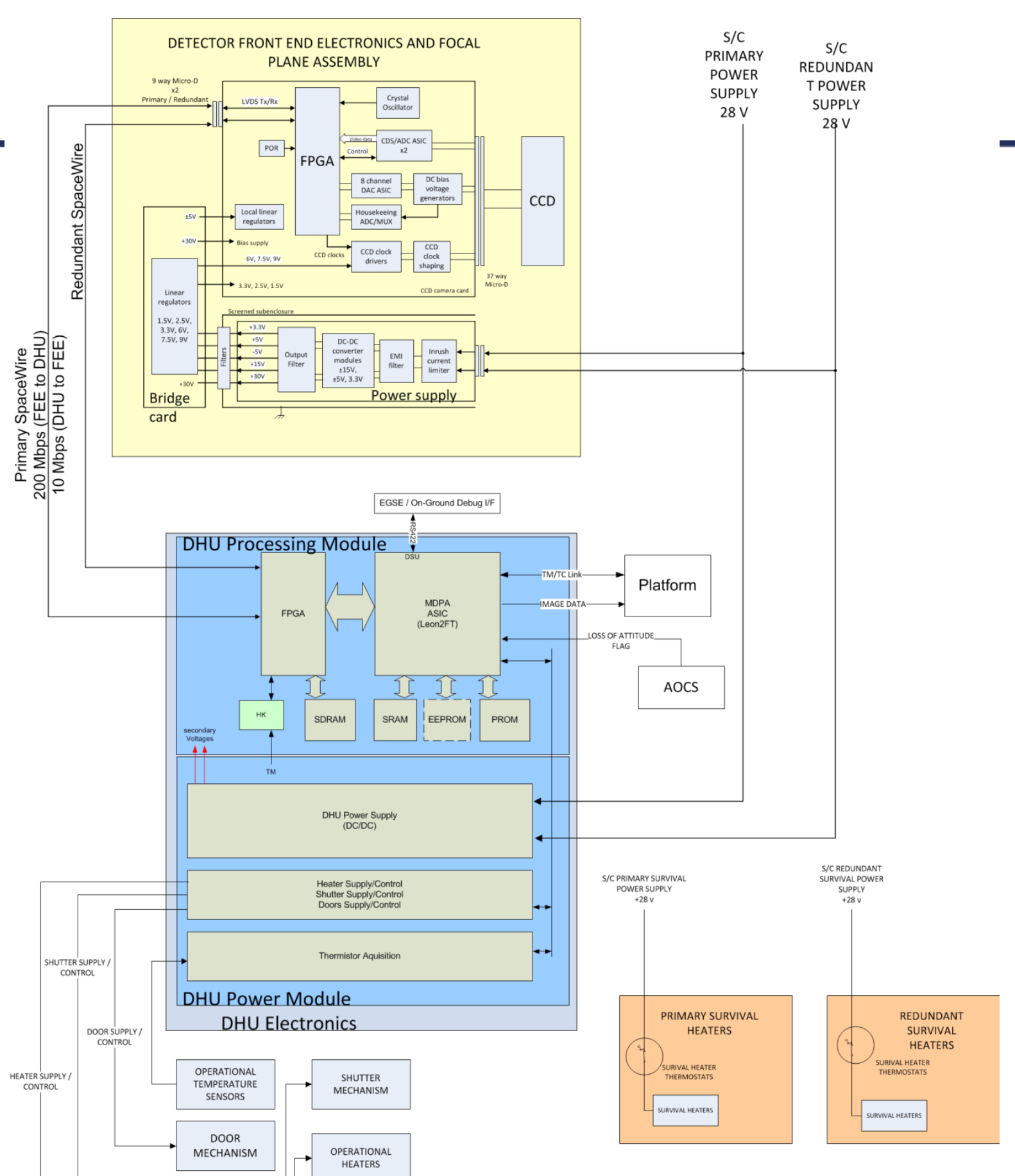




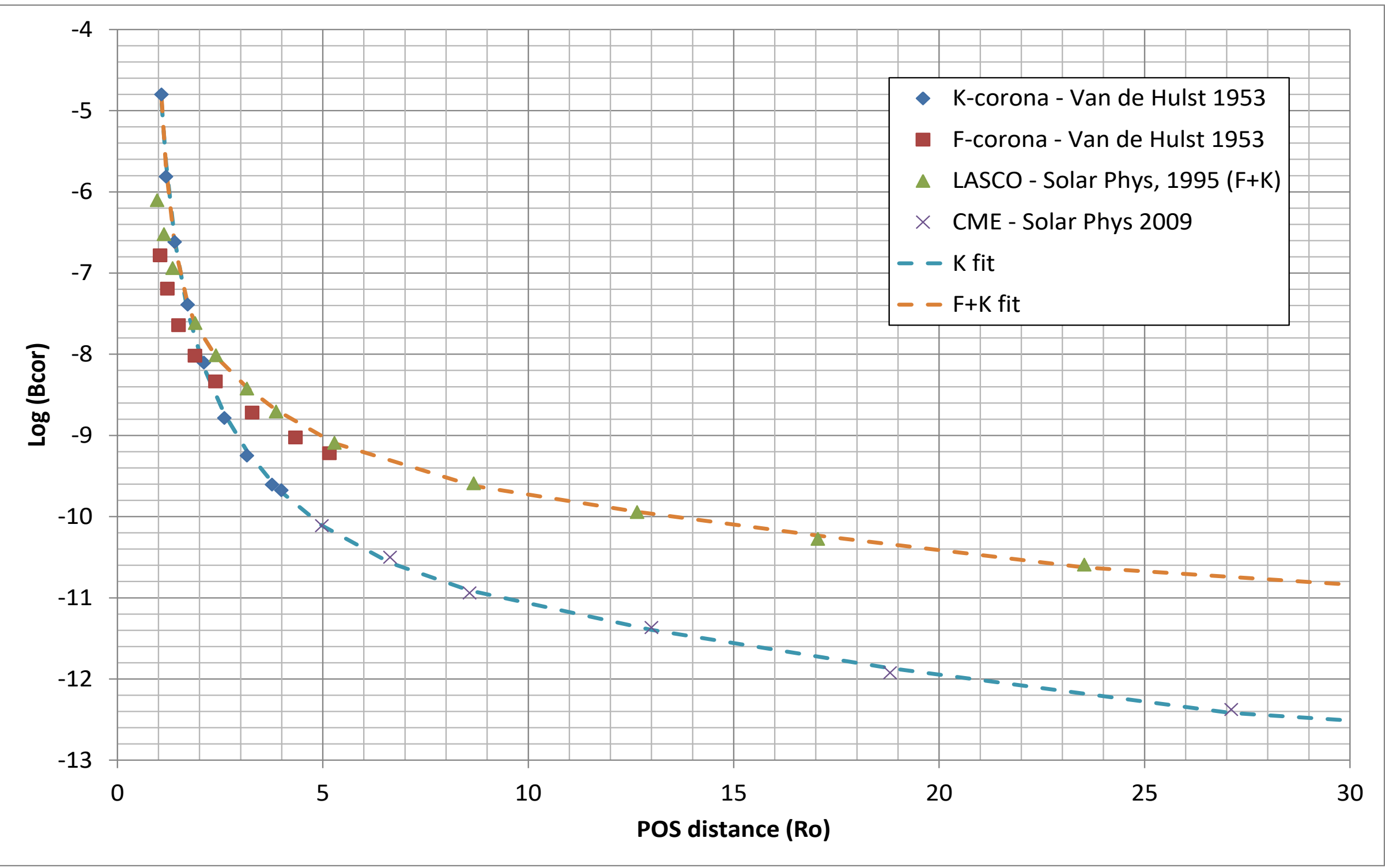


Diffraction model using a modified version of PROPER ${ }^{1}$

(Optical propagation library for IDL)
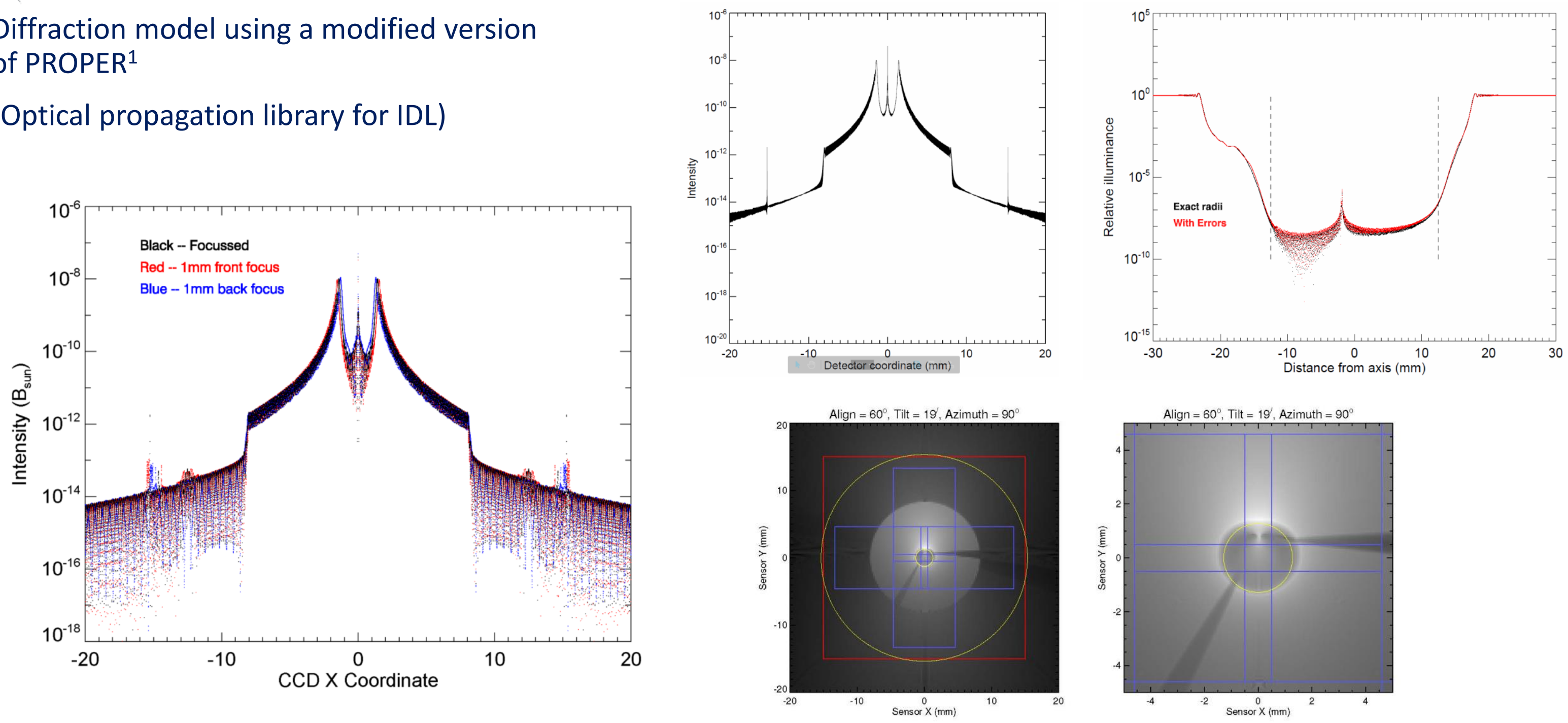

[1] Krist, J. E., "PROPER: an optical propagation library for IDL," Proc. SPIE 6675, (2007) 

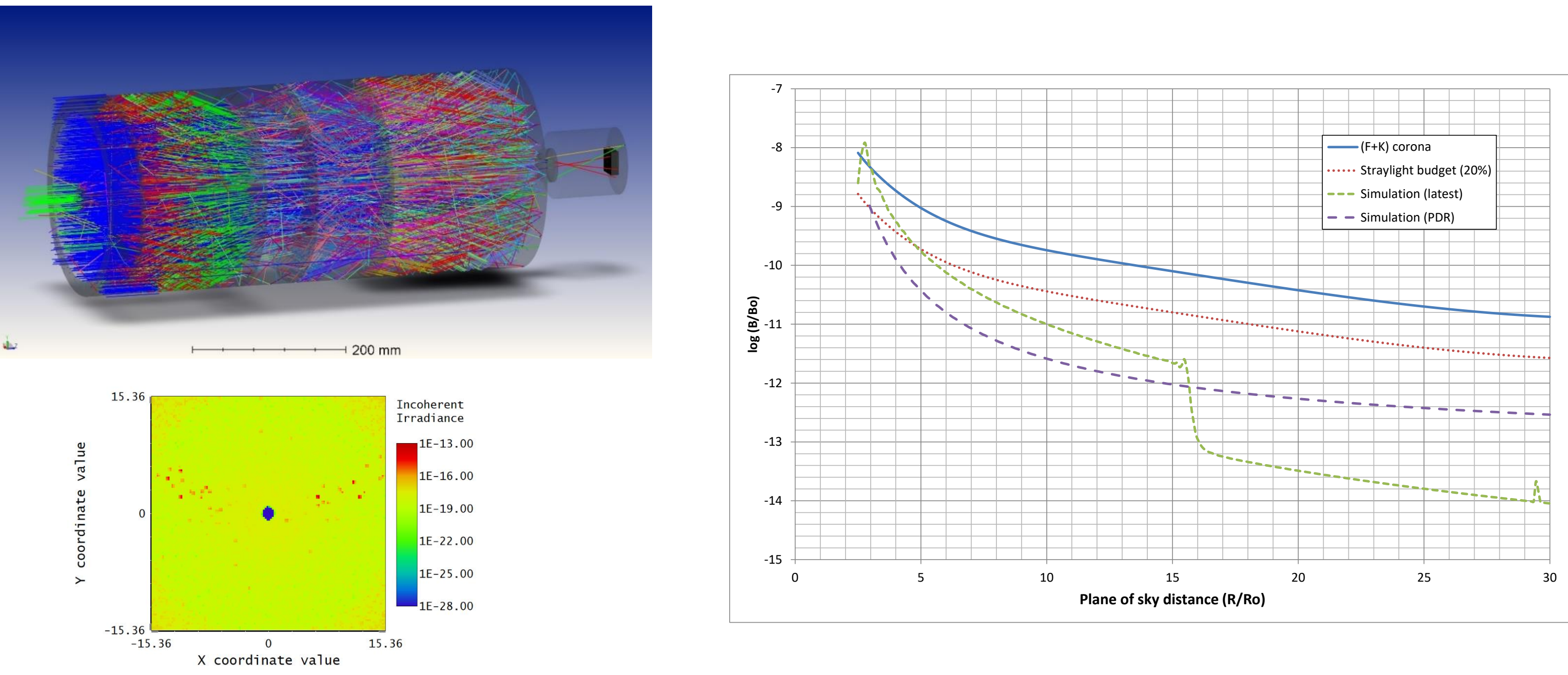

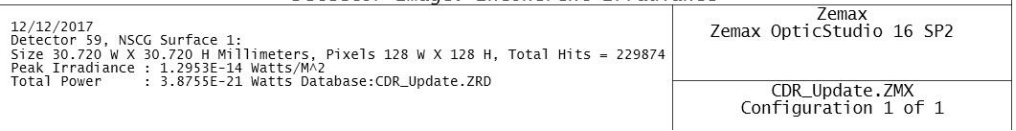



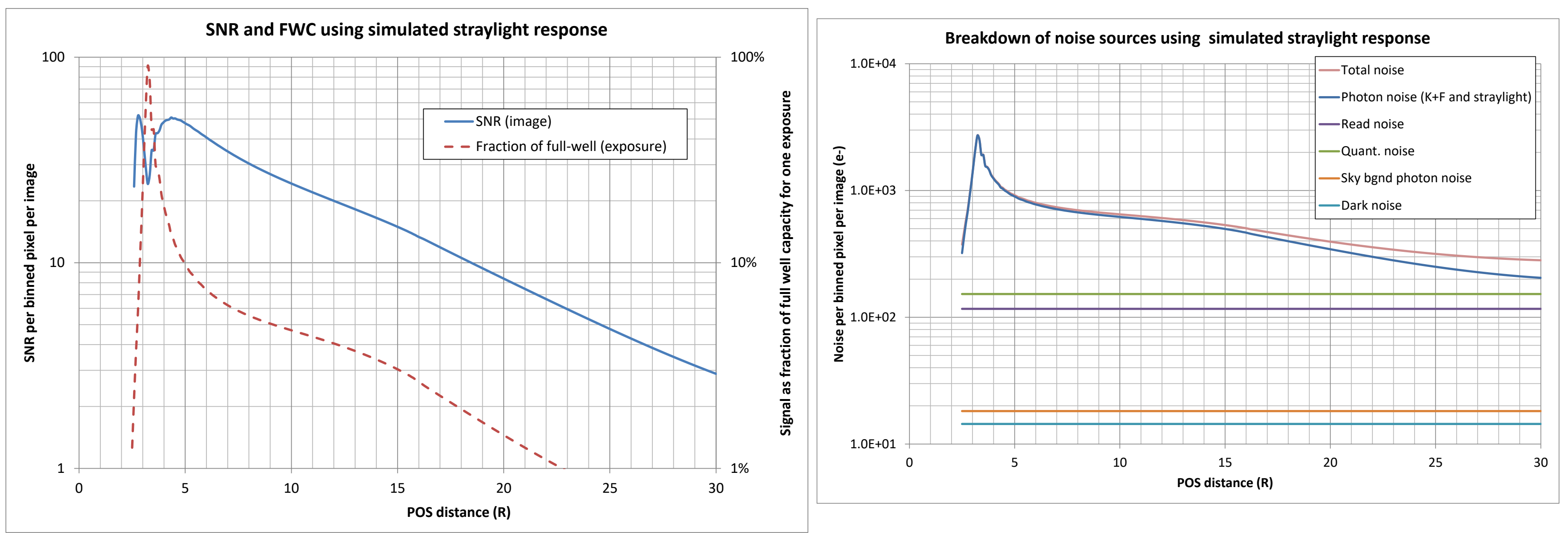

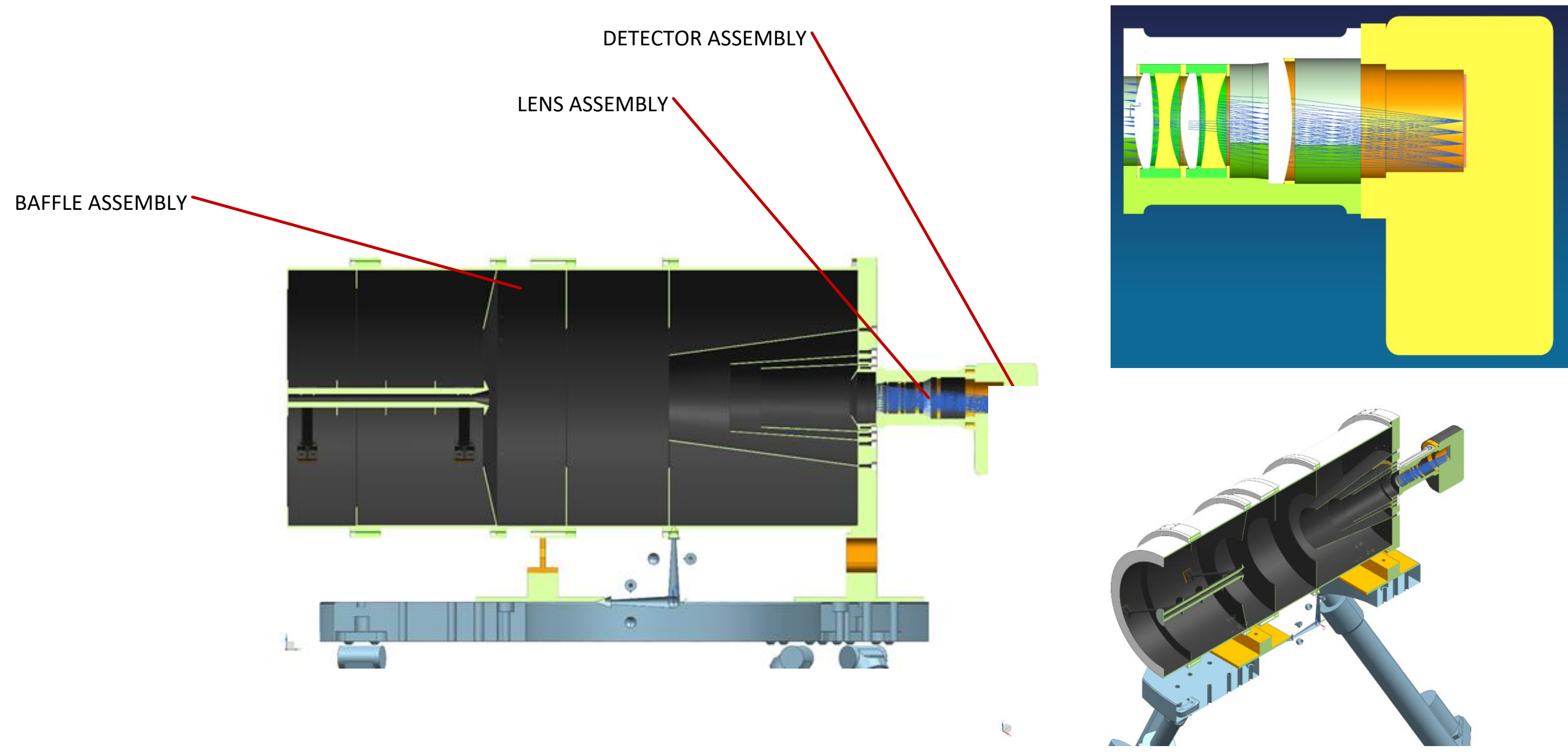
Straylight Testing
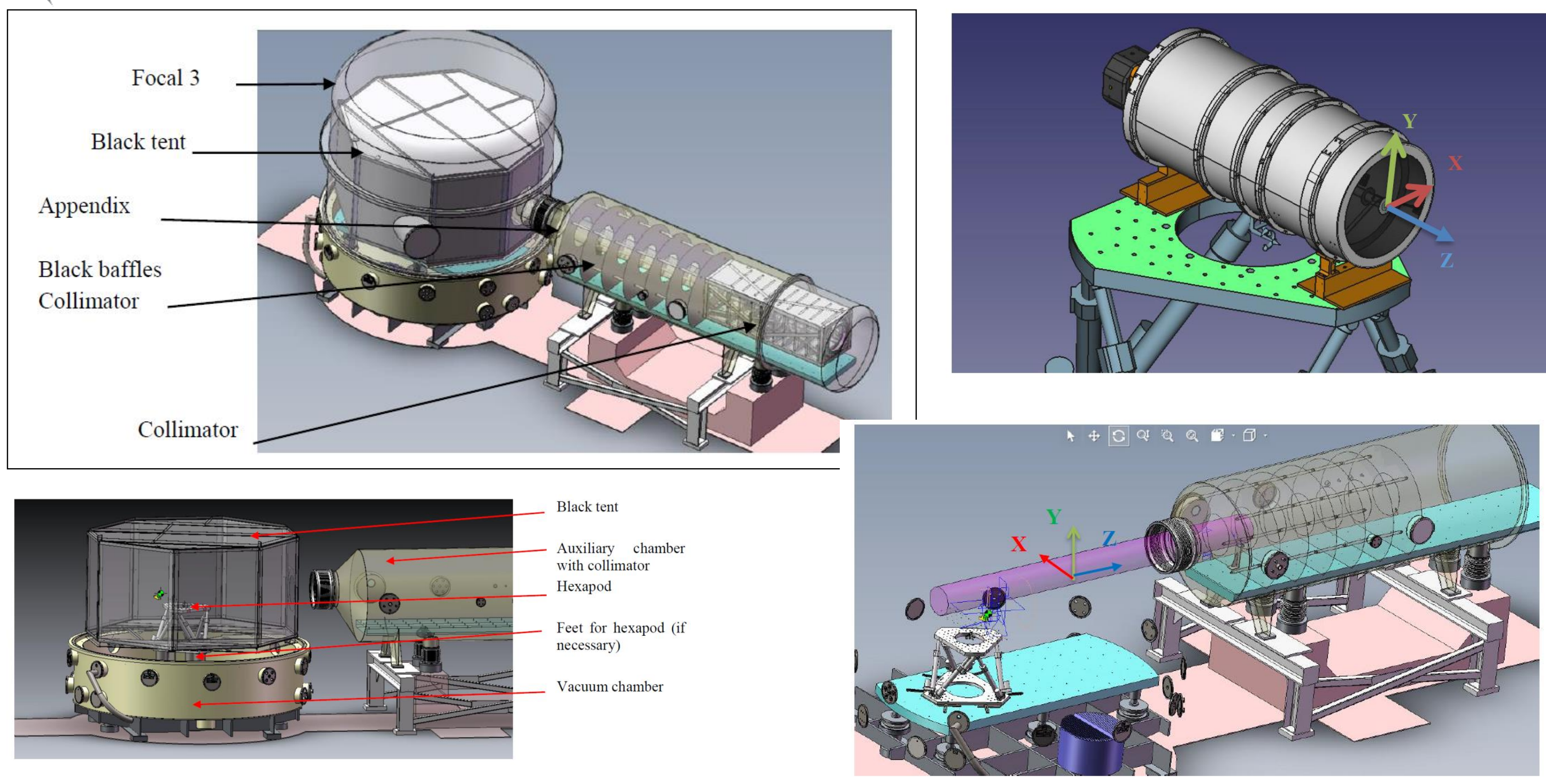


\section{Straylight Testing}
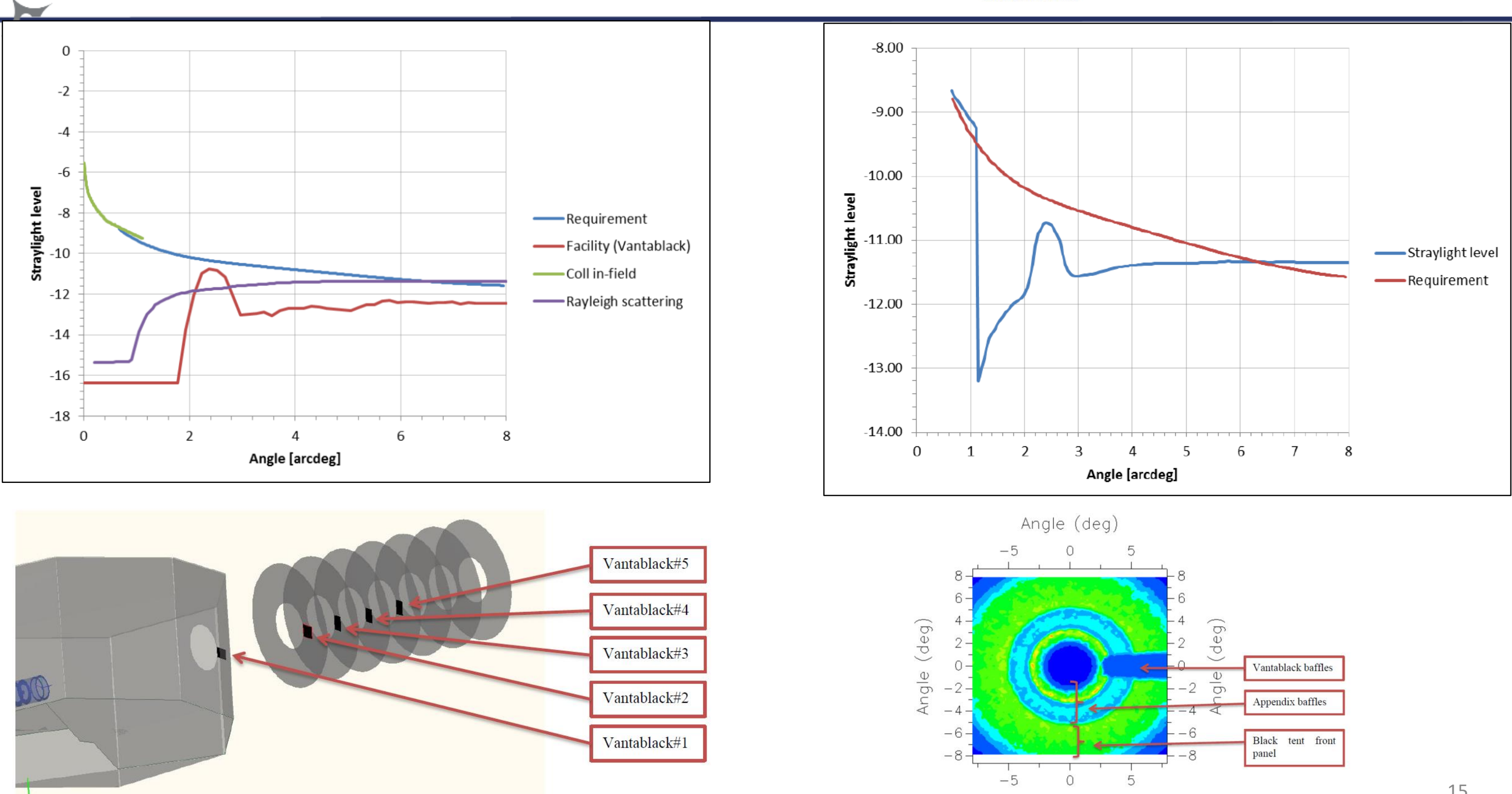

Angle (deg)

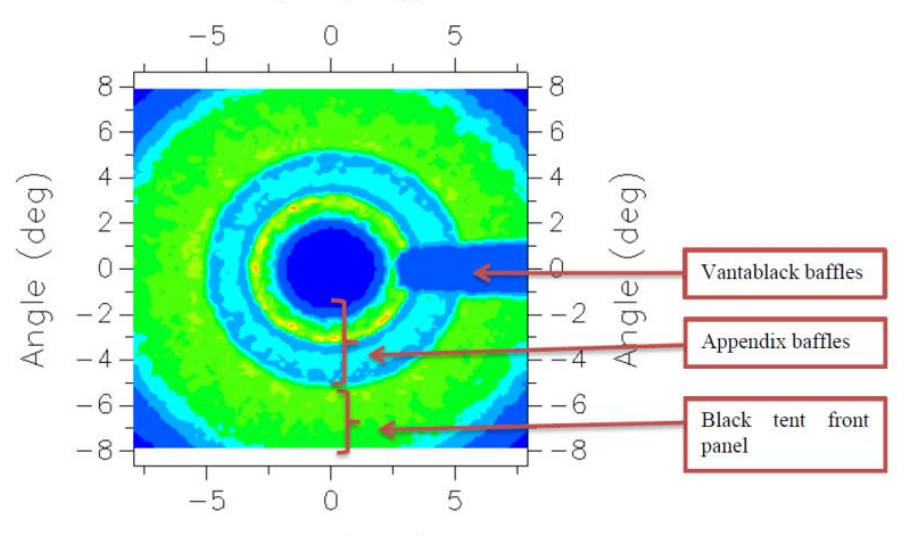




\section{Acknowledgements}

$>$ This work was performed under the ESA Contract No. 4000116072/15/NL/LF as part of ESA's General Support Technology Programme (GSTP)

The SCOPE (Solar Coronagraph for OPErations) consortium:

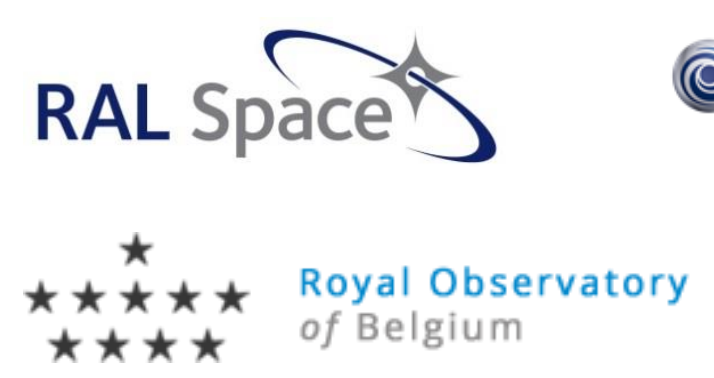

(C) AIRBUS

DEFENCE \& SPACE

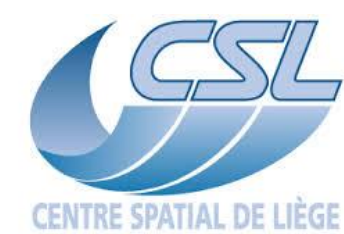

